\title{
No Visible Posterior Motion of Tongue Base During Swallowing
}

National Cancer Institute

\section{Source}

National Cancer Institute. No Visible Posterior Motion of Tongue Base During

Swallowing. NCI Thesaurus. Code C127329.

A finding of no visible posterior motion of tongue base during swallowing. 